\title{
A Patient with An Acute Febrile Illness, Bilateral Pulmonary Infiltrates, Neurologic Symptoms, and a Corpus Callosal Lesion
}

Davis Sam, MD, MHA, Jesse Lu, MD, Michaela Walter, MD, FRCPC

\author{
About the Authors \\ Davis Sam, MD, MHA, Jesse Lu, MD and Michaela Walter, MD, FRCPC are with the Department of Medicine, University of Calgary, \\ Calgary, $A B$ \\ Corresponding Author: mrvwalte@ucalgary.ca \\ Submitted: March 16, 2019. Accepted: April 15, 2019. Published: November 19, 2019. DOI: 10.22374/cjgim.v14i4.360
}

A 45-year-old healthy male presented with sudden onset confusion, slurred speech, and abnormal gait, preceded by 3 days of fever, malaise, and non-productive cough. He had recently traveled to a rural community. Physical examination was notable for a temperature of $40.4^{\circ} \mathrm{C}$, oxygen saturation of $91 \%$ on room air, diffuse bilateral inspiratory crackles on chest auscultation, disorientation to date with some short-term memory impairment, marked dysarthria, upper and lower extremity dysmetria, and truncal ataxia with a wide, ataxic gait. Initial biochemistry was notable for a mild leukocytosis, sodium of $128 \mathrm{mmol} / \mathrm{L}$, and C-reactive protein of $419.3 \mathrm{mg} / \mathrm{L}$. Chest radiography demonstrated bilateral, multilobar, dense airspace consolidation (Figure 1).

The patient was admitted to hospital and magnetic resonance imaging (MRI) with gadolinium enhancement of the brain was performed due to his neurologic symptoms. This revealed a well-circumscribed, ovoid focus of restricted diffusion and faint T2 signal hyperintensity in the splenium of the corpus callosum without any other intracranial abnormality (Figure 2). Urinary antigen testing was positive for Legionella pneumophila serotype 1, while other infectious investigations were negative. He was treated with levofloxacin $750 \mathrm{mg}$ orally daily to complete a 2-week course. His respiratory and neurologic status gradually improved, and he was discharged after 8 days in the hospital.

Legionnaires' disease is a clinical syndrome of atypical pneumonia with systemic manifestations caused by Legionella infection. ${ }^{1}$ While respiratory symptoms are a known feature of the syndrome, the neurologic sequelae are often overlooked by clinicians or cause confusion with alternative diagnoses. ${ }^{2}$ Increased use of MRI has led to the identification of certain signs, such as lesions in the corpus callosum, that may suggest Legionnaires' disease, although thus far these have been limited to several case reports. ${ }^{3}$ Urine antigen testing may be used to confirm infection with Legionella pneumophila serotype $1^{4}$; however, in patients with severe pneumonia, clinicians should still consider the presence of other pathogens before narrowing therapy. Current guidelines for the management of community-acquired pneumonia recommend testing for

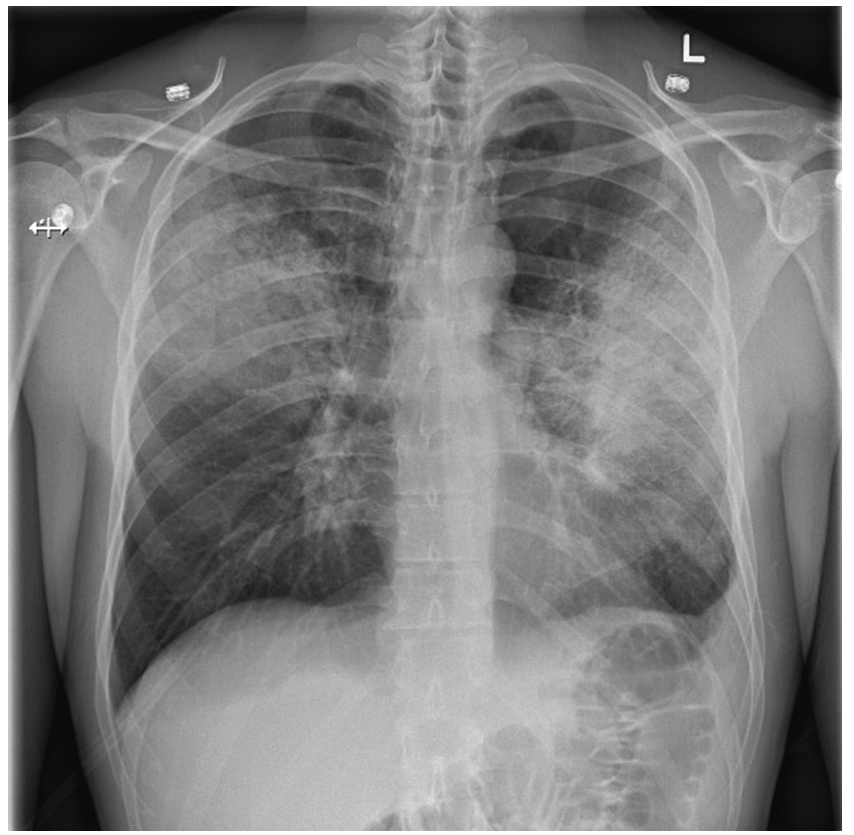

Figure 1. Posteroanterior chest radiograph showing bilateral, multilobar, dense airspace consolidation. 


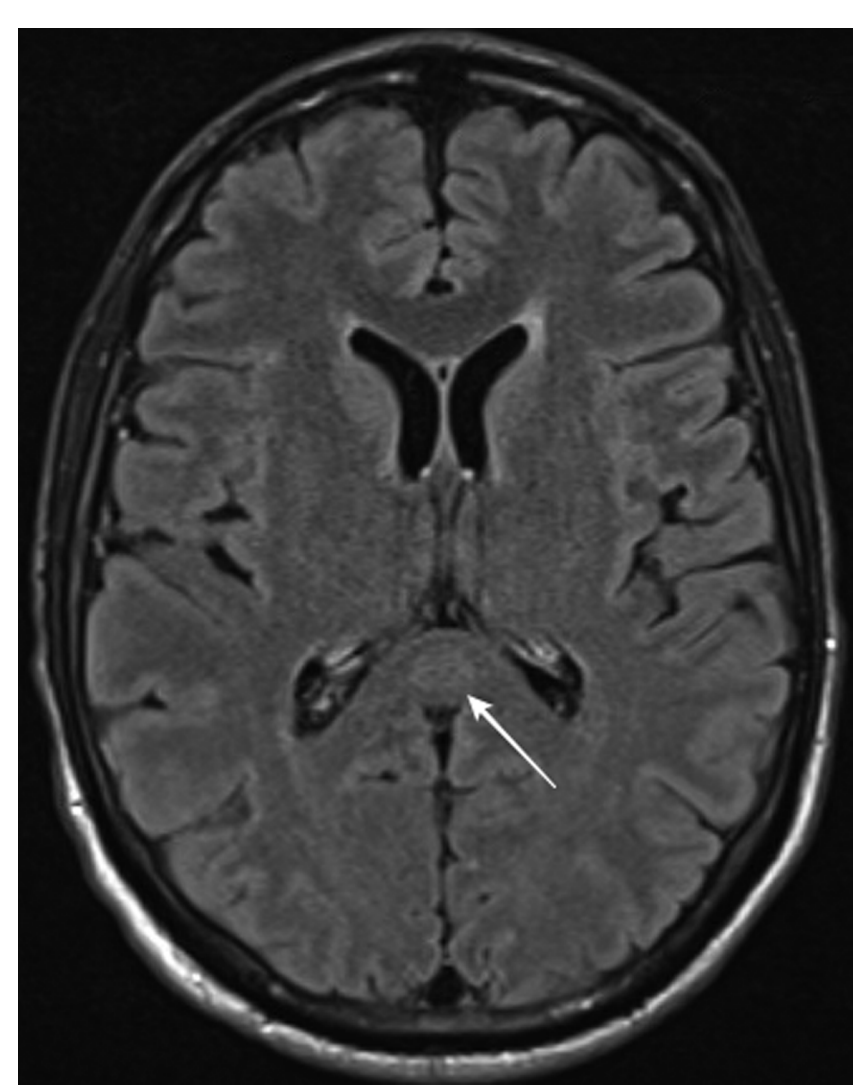

Figure 2. Brain magnetic resonance imaging showing a faint $\mathrm{T} 2$ hyperintensity in the splenium of the corpus callosum (arrow).
Legionella infection in certain patients with communityacquired pneumonia, such as those with severe disease, failure of outpatient therapy, or recent travel. ${ }^{4}$ The preferred treatments are levofloxacin or azithromycin, with duration depending on the severity of presentation. ${ }^{1,4}$ In conclusion, a patient presenting with an acute febrile illness, bilateral pulmonary infiltrates, and neurologic symptoms should be investigated for Legionnaires' disease.

\section{Disclosures}

The authors have nothing to disclose.

\section{Funding}

None.

\section{References}

1. Cunha BA, Burillo A, Bouza E. Legionnaires' disease. Lancet 2016;387:376-85.

2. Morgan JC, Cavaliere R, Juel VC. Reversible corpus callosum lesion in legionnaires' disease. J Neurol Neurosurg Psychiatr 2004;75:651-4.

3. Tomizawa Y, Hoshino Y, Sasaki F, et al. Diagnostic utility of splenial lesions in a case of Legionnaires' disease due to Legionella pneumophila serogroup 2. Intern Med 2015;54:3079-82.

4. Mandell LA, Wunderink RG, Anzueto A, et al. Infectious Diseases Society of America/American Thoracic Society consensus guidelines on the management of community-acquired pneumonia in adults. Clin Infect Dis 2007;44 Suppl 2:S27-72. 